\title{
Long-term outcomes of patients with myopic traction maculopathy after phacoemulsification for incident cataract
}

\author{
Lei Cai ${ }^{1,2,3} \cdot$ Zhongcui Sun ${ }^{1,2,3} \cdot$ Diwen Guo ${ }^{4}$ Qi Fan ${ }^{1,2,3} \cdot$ Xiangjia Zhu' ${ }^{1,2,3} \cdot$ Jin Yang $^{1,2,3} \cdot$ Yi Lu $^{1,2,3}$
}

Received: 25 September 2018 / Revised: 27 December 2018 / Accepted: 8 March 2019 / Published online: 5 April 2019

(c) The Royal College of Ophthalmologists 2019

\begin{abstract}
Purpose To evaluate the long-term effect of cataract surgery on highly myopic patients with myopic traction maculopathy (MTM) and the risk factors associated with MTM progression.

Methods Highly myopic patients with cataract and MTM were included. Phacoemulsification surgery was performed on patients who had vision loss below 20/63 and were willing to operation. Exclusion criteria included full thickness macular hole, foveal/retinal detachment, history of vitreoretinal surgery, myopic choroidal neovascularization, macular chorioretinal atrophy, peripheral lattice degeneration, incomplete follow up, or intraoperative complications. All patients underwent a complete ophthalmological examination. Optical coherence tomography examinations and microperimetry examinations were performed.

Results A total of 229 patients (mean age: $57 \pm 6$ years) were recruited, including 179 operated patients and 50 unoperated patients. Both the best corrected visual acuity (BCVA) and macular sensitivity (MS) were significantly improved after cataract surgeries throughout the follow-up period $(p=0.000)$. No difference was found in the proportion of MTM staging and in the rate of resolving/stable or progressive MTM $(p=0.757)$ between the operated and the unoperated groups. Of all patients, those with S2 to S4 MTM at baseline had significantly higher risk of progressive MTM $(p<0.001)$. Patients with absence of posterior vitreous detachment or with longer axial length at baseline had higher risks of progressive MTM.

Conclusion Cataract surgery generally improves the BCVA and MS of highly myopic patients with MTM. Preoperative vitreoretinal adhesion, longer axial length, and S2 to S4 MTM are risk factors for progressive MTM. A long-term follow-up on the development of MTM is recommended.
\end{abstract}

These authors contributed equally: Lei Cai, Zhongcui Sun

These authors contributed equally: Jin Yang, Yi Lu

Jin Yang

jin_er76@hotmail.com

$\triangle \mathrm{Yi} \mathrm{Lu}$

luyieent@126.com

1 Department of Ophthalmology, Eye \& ENT Hospital of Fudan University, 83 Fenyang Road, 200031 Shanghai, China

2 Key NHC Key Laboratory of Mopia (Fudan University), Laboratory of Myopia, Chinese Academy of Medical Sciences, 200031 Shanghai, China

3 Shanghai Key Laboratory of Visual Impairment and Restoration, 200031 Shanghai, China

4 Department of Ophthalmology, Tongren Hospital, Shanghai Jiaotong University School of Medicine, 1111 Xianxia Road, 200336 Shanghai, China

\section{Introduction}

High myopia is one of the most common diseases causing social blindness, especially in East Asian countries [1,2]. High myopia is characterized by refractive error exceeding -6.0 spherical diopters (DS) and axial length elongation over $26.5 \mathrm{~mm}$ [3], and induces various specific complications [4], including cataract formation [5] and myopic traction maculopathy (MTM). MTM affects $9-34 \%$ of highly myopic eyes with posterior staphyloma and is composed of a series of pathological conditions including vitreomacular traction, retinal thickening, macular retinoschisis, lamellar macular hole, and foveal retinal detachment [6-8]. MTM is well diagnosed using optical coherence tomography (OCT) [9].

In highly myopic patients, both MTM and incident cataract cause visual impairment. Vitrectomies were reported to be useful in treating MTM [10, 11]. However, vitrectomies could also lead to serious complications [12, 13]. 
Moreover, patients with MTM might have fairly stable visual function and macular structures for long time [8, 14], and MTM was also reported to resolved spontaneously [15]. These results suggested that vitrectomy might be not definitely beneficial or necessary for patients with MTM.

Cataracts in highly myopic patients appear at earlier age and progress rapidly [1]. Cataract surgery might be effective in improving visual function despite of MTM. Some researchers suggested that MTM exacerbated after cataract surgery [16-18], while some others believed that cataract extraction improved visual function despite of postoperative increased central foveal thickness [19]. However, these studies contain small series of patients and the follow-up period is limited. In this study, we performed phacoemulsification on 179 highly myopic patients with MTM and evaluated the long-term (36 months) prognosis of macular structures and visual acuity after cataract surgery.

\section{Methods}

\section{Subjects}

This is a prospective study of 229 consecutive highly myopic patients with cataract and MTM seen at the Eye and Ears, Nose, and Throat (ENT) Hospital, Fudan University, between March 2012 and December 2017 (sample size was estimated on the basis of similar research reported in previous studies [20]). Exclusion criteria included full thickness macular hole (FTMH), foveal/retinal detachment, history of vitreoretinal surgery, myopic choroidal neovascularization (CNV), macular chorioretinal atrophy [11,21, 22], peripheral lattice degeneration, loss to follow-up or follow-up time $<36$ months, or intraoperative posterior capsular rupture. All patients underwent a complete ophthalmological examination including best corrected visual acuity (BCVA) using Snellen chart, slit-lamp biomicroscopy, indirect ophthalmoscopy with dilation of pupils, intraocular pressure, and color photography. Structures of the posterior retina and posterior vitreous were examined using OCT and B-scans. Axial lengths were measured using IOLMaster 500 (Carl Zeiss, Germany). Patients who underwent cataract surgery also had the microperimetry exam. This study was approved by the Ethic Committee of Eye and ENT Hospital, Fudan University, Shanghai, China, and was conducted in compliance with the Declaration of Helsinki. This study was registered with ClinicalTrials.gov, number NCT03062085. Written informed consent was obtained from all patients.

Phacoemulsification surgery was performed on patients who had vision loss below 20/63 and were willing to the operation. Situations that cataract surgery was not performed included: (1) patients refused the surgery, (2) cataract was mild and did not impair visual acuity significantly; or (3) fundus examination suggested severe MTM, not cataract, to be the major cause of vision loss. All patients were advised to have immediate examinations in case of visual impairment or metamorphopsia. The operated patients were examined days before surgery and 3months, 12 months, 24 months, and 36 months after surgery. Unoperated patients were followed up at baseline, 3 months, 12 months, 24 months, and 36 months. If cataract became severe during follow ups and removal of cataract was considered beneficial to vision, cataract surgery was advised and performed on those who accepted the surgery. These patients would have another 36-months' postoperative follow ups.

\section{OCT examination}

According to previous works [23], the Cirrus HD-OCT scans (Cirrus HD-OCT, Carl Zeiss Meditec, Dublin, CA) were performed using macular cubes and 5-line raster scanning protocols to evaluate the posterior retina. According to Shimada et al. [20], we subdivided the macular features of our patients into 5 stages ( $\mathrm{S} 0$ to $\mathrm{S} 4$ ): S0, no macular retinoschisis; S1, extrafoveal macular retinoschisis; S2, fovea-only macular retinoschisis; S3, both foveal and extrafoveal macular retinoschisis, but not the entire macula; and S4, retinoschisis in the entire macular area (Fig. 1). After cataract surgery, a decrease in the extent of schisis cavities indicates resolution of MTM. Any of the following features indicates progression of MTM: increase in height $(>100 \mu \mathrm{m})$ or/and width of schisis cavities, development of FTMH or LMH, or foveal retinal detachment. Eyes that did not meet the criteria for MTM resolution or progression were stable MTM.

\section{Microperimetry examination}

We performed microperimetry on subjects who had cataract surgery, dilated with $1 \%$ tropicamide, with a perimeter (Nidek MP-1, Nidek Technologies, Padova, Italy). We then trained the subjects to fixate a target and respond correctly to the stimuli. Subsequently, eye movement of subjects was tracked automatically by an eye-tracking system with the capacity to calculate a subtle eye shift relative to a distinct fundus marker (major vessels or optic nerve head). In the automated pattern, each exam consisted of a 4-2-1 staircase strategy on a series of 45 Goldmann III stimuli covering the central $12^{\circ}$ of visual field. Intensity of each stimulus varied at $1(0.1 \log )$ step scale from 0 to 20 decibels (dB). Answers of the subjects were recorded and all stimuli were displayed on the same, 

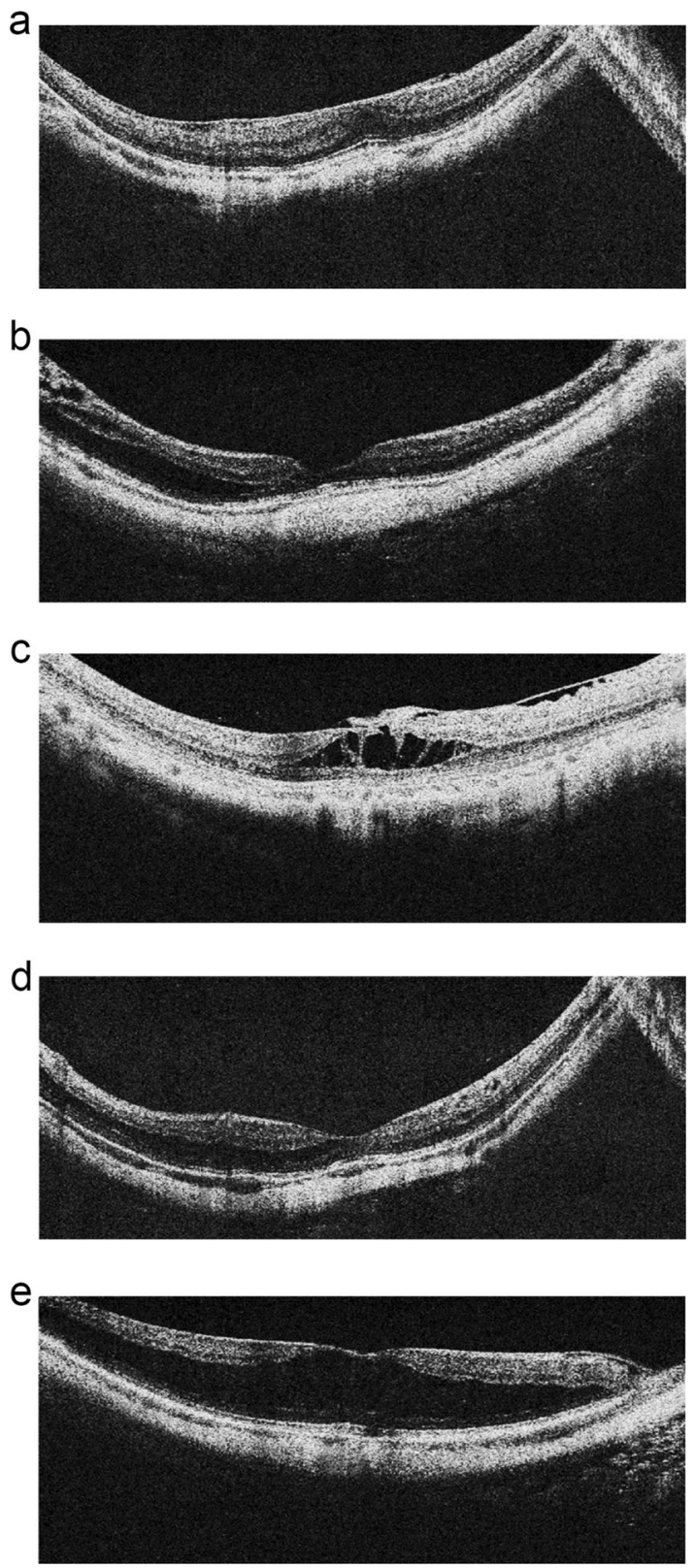

Fig. 1 Classification of Myopic Traction Maculopathy. a S0, no macular retinoschisis; b S1, extrafoveal macular retinoschisis; c S2, foveal but not the entire macular area macular retinoschisis; d S3, both fovea and extrafovea but not the entire macula; and e $\mathrm{S} 4$, retinoschisis in the entire macular area

fixed, infrared fundus image acquired at the beginning of the examination [24].

A false-positive test stimulus was projected periodically every $60 \mathrm{~s}$ onto the optic disk. Duration of each test was limited within $15 \mathrm{~min}$ to avoid fatigue. Functional results were displayed on a color digital retinograph - a vivid fusion of microperimetric data and the corresponding retina. The documented macular sensitivity (MS) was the mean value of the collected data from 45 stimuli during the test.

\section{Surgical procedure}

All of the phacoemulsification was performed by a single skilled cataract surgeon (Dr. Yi Lu) using the INFINITI Vision System (Alcon Inc., Fort Worth, TX, USA). In brief, a $2.4-2.6 \mathrm{~mm}$ self-sealing clear corneal incision was fashioned after topical anesthesia, followed by continuous capsulorhexis, hydrodisection, phacoemulsification, and manual aspiration of the cortex with a balanced salt solution. A foldable intraocular lens (IOL, Superflex Aspheric 970 C/920 H, Rayner, UK) was implanted into the capsular bag. The incision was watertight without suturing Postoperative prescription included topical steroid (1\% prednisolone acetate at 4 times per day for 2 weeks and at 3 times daily for another week), topical nonsteroidal antiinflammatory drug $(0.1 \%$ pranoprofen for 4 weeks at 4 times per day) and antibiotic eye drop ( $0.5 \%$ levofloxacin for 3 weeks at 4 times per day).

\section{Statistical analysis}

Statistical analyses were performed using SPSS 20.0 (IBM Corp, Armonk, NY). Mann-Whitney $U$ test was used for comparisons. $\chi^{2}$ test and Fisher exact probability test were used to seek the risk factors for the progression of MTM. Two-tailed Student's $t$-test for continuous variables [25] and $\chi^{2}$ test for categorical variables were used to compare features of MTM between two groups. Values were reported as mean \pm stardard deviation $(\mathrm{SD})$. Differences were considered significant at $P$ value $<0.05$.

\section{Results}

\section{Clinical characteristics of patients with MTM}

A total of 229 patients (mean age: $57 \pm 6$ years) with MTM diagnosed by OCT were recruited in this study, including 98 males and 131 females. Totally 179 patients underwent cataract extraction and 50 patients did not. No difference was found between operated and unoperated patients in age, sex, axial length, and proportion of subgroups. At baseline, the operated patients had worse visual acuity $(p=0.000)$ and higher refractive error $(p=0.000)$ than the unoperated patients. Patients' demographics features wee summarized in Table 1.

Cataract surgeries improved visual acuities and MS in all operated patients (Fig. 2). Preoperative BCVA was 20/160 \pm $20 / 200$, and preoperative MS was $7.3 \pm 2.4 \mathrm{~dB}$. The visual acuity was stable at the end of follow up (Fig. 2a). Postoperative visual acuities of the operated patients were significantly better than the unoperated patients $(p=0.000)$. Forty-three patients underwent Yttrium aluminium garnet 
Table 1 Patient demographics and grouping

\begin{tabular}{lllr}
\hline & Operated group & Control group & $P$ value \\
\hline Age (years) & $56 \pm 6$ & $58 \pm 6$ & 0.994 \\
Sex (male/female) & $84 / 95$ & $22 / 28$ & 0.750 \\
Axial length (mm) & $29.7 \pm 1.5$ & $29.4 \pm 1.3$ & 0.990 \\
Refractive errors (DS) & $-15.4 \pm 3.1$ & $-13.4 \pm 3.2$ & $<0.001$ \\
BCVA (base.) & $20 / 160 \pm 20 / 200$ & $20 / 32 \pm 20 / 160$ & $<0.001$ \\
BCVA (1 y.) & $20 / 32 \pm 20 / 100$ & $20 / 32 \pm 20 / 160$ & \\
BCVA (Fnll.) & $20 / 32 \pm 20 / 100$ & $20 / 40 \pm 20 / 200$ & $<0.001$ \\
MS (dB) (base.) & $7.3 \pm 2.4$ & $/$ & \\
MS (dB) (1 y.) & $14.7 \pm 2.3$ & $/$ & \\
MS (dB) (Fnll.) & $13.7 \pm 2.8$ & $/$ & \\
Grouping & & & \\
S0 & 31 & 7 & \\
S1 & 38 & 12 & \\
S2 & 29 & 12 & \\
S3 & 46 & 11 & \\
S4 & 35 & 8 & \\
Changes of OCT & & 45 & \\
finding during the & & & \\
follow-up period & & & \\
Improved & 162 & & \\
Stable & 4 & & \\
Progressed & 13 & & \\
\hline
\end{tabular}

base. baseline, $1 y 1$ year, Fnll. final, DS Spherical diopters, BCVA best corrected visual acuity, $\mathrm{mm}$ millimeter, yrs. years old.

(YAG) laser capsulotomies at 24 months (15 patient) and at 36 months (28 patients) and their visual acuities all recovered to the level of the first postoperative visit. Notably, we found that the MS values at 3 years postoperatively were subtly declined in comparison with the values at 1 year postoperatively, even after the YAG laser capsulotomy (Fig. 2b-d). BCVA of the unoperated patients remained stable as far as 36 months.

\section{Development of MTM}

Based on macular features on OCT, MTM were subdivided into 5 stages: S0, S1, S2, S3, and S4 (See Methods). In the operated group, numbers of patients with S0 to S4 MTM at baseline were $31,38,29,46$, and 35 . At the last visit, the numbers were $32,38,26,45$, and 38 , respectively. Compared with baseline status, MTM at the last visit resolved in 4 patients (2.2\%; Fig. 3a, b) and remained stable in 162 patients $(90.5 \%)$ (Fig. 3d). Thirteen patients $(7.3 \%)$ had progressed MTM at the last visit (Fig. 3c), the BCVA and MS of which were dramatically impaired at the final visit as compared with the test at 1 year/postoperatively. Among them, three patients had S2 MTM at baseline (10.3\%, 3/29), four had S3 MTM $(8.7 \%, 4 / 46)$, and six had S4 MTM
$(17.1 \%, 6 / 36)$, respectively. Patients with preoperative S4 MTM had significantly higher risk of progressive MTM than those with lower stages of MTM $(p=0.013)$. There were two patients progressed within 6 months after cataract surgery. One developed FTMH and underwent vitreoretinal surgery. Another patient experienced a significant increase in height and width of schisis cavities in 6 months after cataract surgery, and remained stable afterwards. At the last visit of 36 months, one patient developed FTMH and foveal detachment and underwent vitreoretinal surgery (Fig. 3c).

In the unoperated group, numbers of patients with S0 to S4 MTM at baseline were $7,12,12,11$, and 8 . At the last visit, the numbers were $7,12,13,10$, and 8 , respectively. Compared with baseline status, MTM at the last visit resolved in two patients (4.0\%) and remained stable in 45 patients $(90.0 \%)$. Three patients $(6.0 \%)$ had progressed MTM at the last visit. Among them, one patient had S3 baseline MTM $(9.1 \%, 1 / 11)$ and two had S4 $(25.0 \%, 2 / 8)$. No difference was found in the risk of progressive MTM between the patients with S3 and S4 MTM at baseline ( $p=$ 0.107).

No difference was found in the proportion of MTM staging and in the rate of resolving/stable or progressive MTM $(p=0.757)$ between the operated and the unoperated groups.

\section{Risk factors of progressive MTM}

To evaluate potential risk factors of MTM progression, we compared the differences in age, gender, baseline BCVA, axial length, diopter, posterior vitreous detachment (PVD), and staging between patients with stable/resolved MTM and progressive MTM at the last visit (Fig. 4). Of all the 229 patients, preexisting PVD at baseline was found in 139 patients and $5(3.6 \%)$ of them had MTM progression. PVD was absent in 90 patients and 11 (12.2\%) of them had MTM progression. Patients with PVD at baseline had significantly lower risks of MTM progression $(p=0.017)$.

Of all the patients, numbers of patients with S0 to S4 MTM at baseline were 38, 50, 41, 57, and 43. Numbers of patients with progressive MTM at last visit were 0, 0, 3, 5, and 8 , respectively. Patients with baseline MTM stage 2-4 had significantly higher risk of MTM progression than those with MTM stage 0 and $1(p<0.001)$. No difference was found in the risk of MTM progression between S2 and S3 $(p=1.000)$, between S3 and S4 $(p=0.229)$, or between S2 and S4 $(p=0.196)$.

Another risk factor of progressive MTM was axial length (Fig. 4b). Patients with MTM progression at last visit had significantly longer axial length than those with stable/ resolved MTM (Mann-Whitney $U$ test, $p=0.017$ ).

No difference was found in age, gender, baseline BCVA, and baseline diopter between patients with progressive 
a

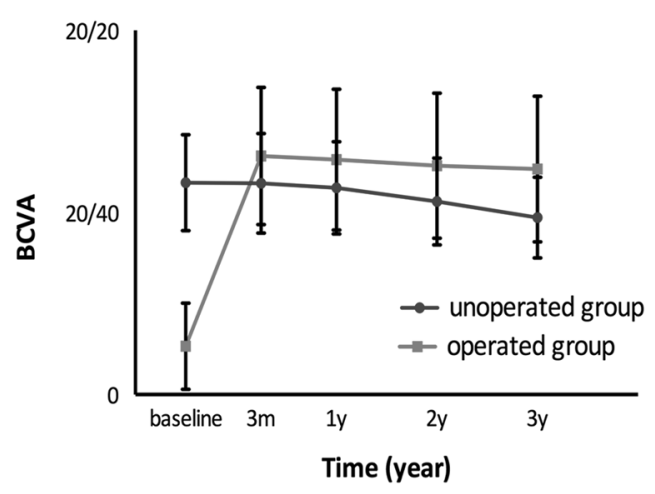

C

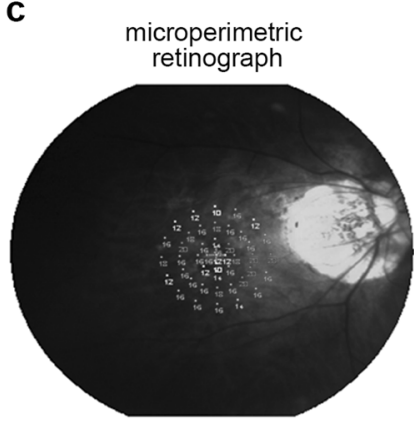

d

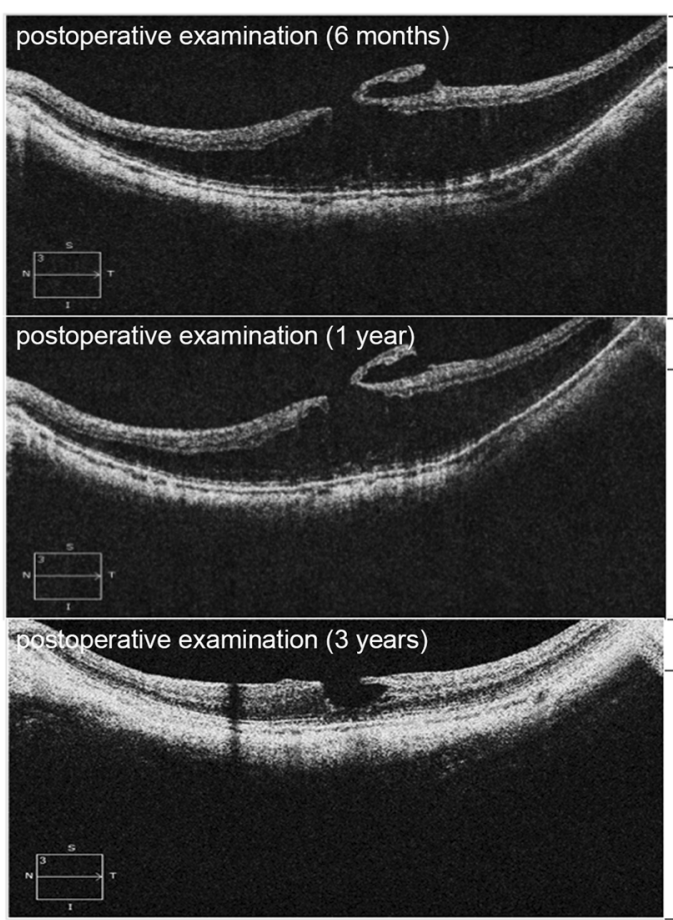

Fig. 2 The changes of BCVA and MS for MTM patients. a The temporal dynamic of BCVA shows a significant improvement of visual acuity after cataract surgery during the 3-year follow-up. BCVA of the unoperated patients remained stable as far as 3 years. $\mathbf{b}$ The statistical analysis of MS shows an increased retinal sensitivity after cataract surgery, although the MS is subtly declined at 3 years postoperatively as compared with the results collected at 1 year b
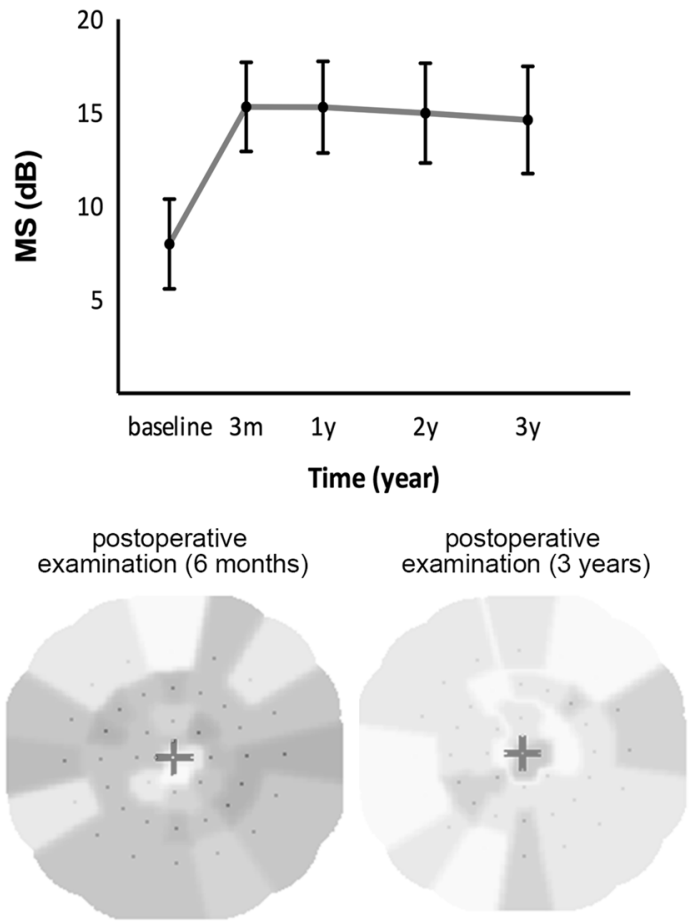

postoperatively. $\mathbf{c}$ Shown is the digital retinography of a representative subject (58 years old at the initial examination). Her macular sensitivity conspicuously increases at 6 months postoperatively but subtly declines at 3 years after surgery. d Shown are the OCT images and digital retinography of left eye in a 66-year-old man at 1,2 , and 3 years postoperatively. The height of retinoschisis is strikingly reduced but the MS is not changed at the final visit 
a

postoperative examination (3 months)

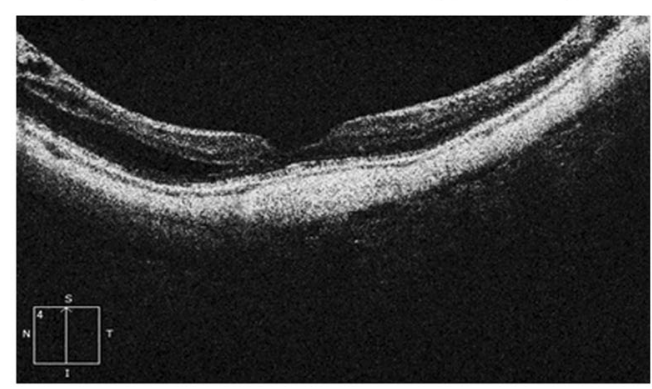

b

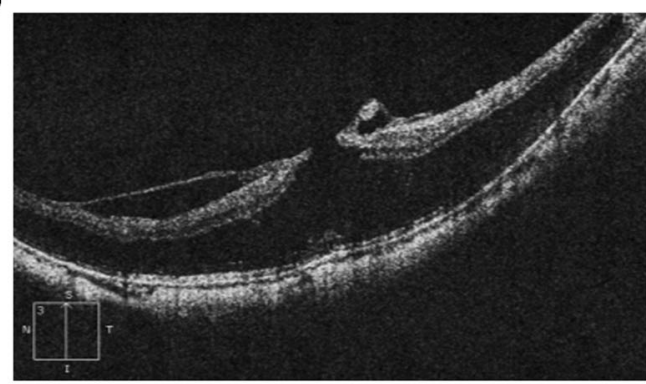

C

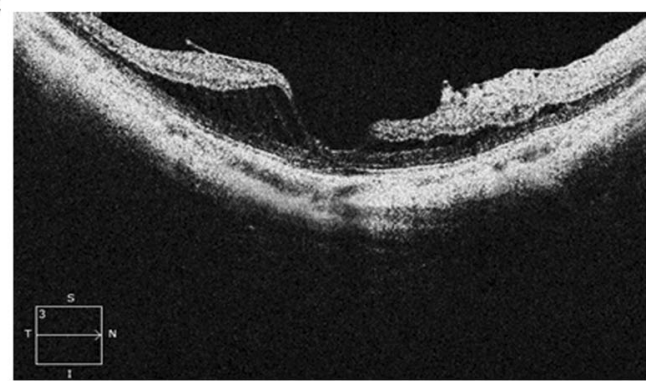

d

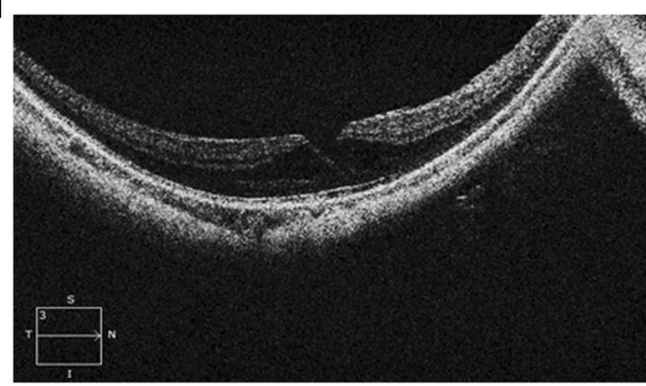

e

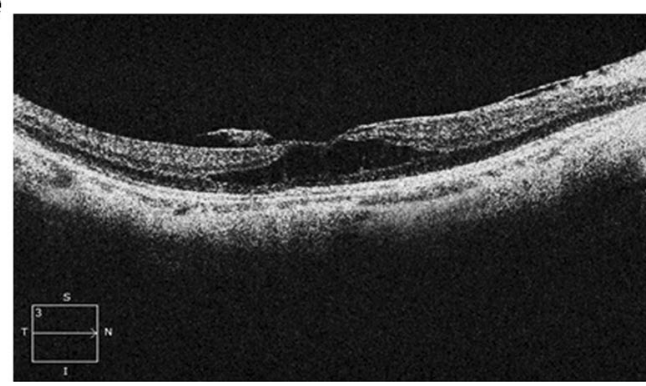

postoperative examination (3 years)
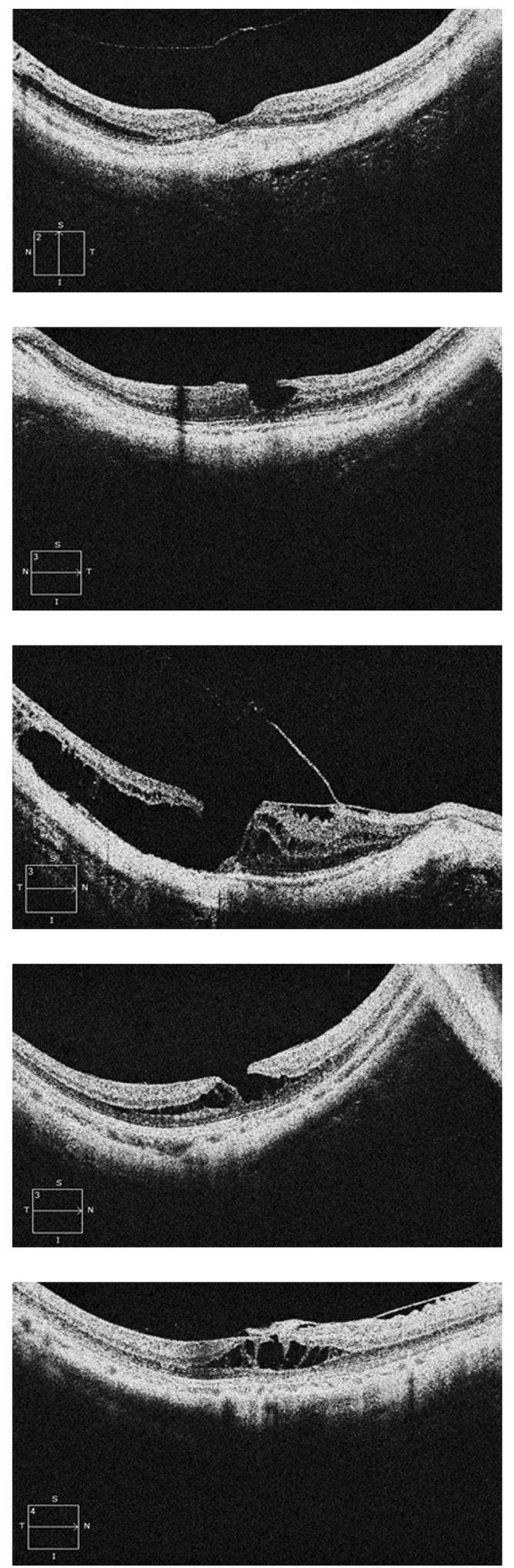

MTM and stable/resolved MTM. No difference was found in the rate of resolving/stable or progressive MTM ( $p=$ 0.757 ) between the operated and the unoperated groups.
The nuclear opalescence (NO), the average cumulated dissipated energy (CDE) and the mean ultrasound time of operated patients were documented and compared. No 
Fig. 3 Examples of Patients with Resolved, Stable and Progressive Myopic Traction Maculopathy (MTM). a Optic coherent tomography (OCT) images of the left eye of a 62-year-old male patient showed small macular retinoschisis inferior to the fovea at baseline (S1 MTM). Posterior vitreous detachment (PVD) was not found. At the follow up of 36 months, complete PVD occurred and the schisis cavities resolved to S0 MTM. b OCT images of the left eye of a 66-year-old male patient showed an extensive macular retinoschisis before cataract surgery (S4 MTM). At the final visit, macular retinoschisis resolved to S0 MTM. c OCT images of the right eye of a 55-year-old female patient showed extensive macular retinoschisis at baseline (S4 MTM). Three years later, the maculopathy progressed to a full-thickness macular hole with foveal detachment. She was advised and performed vitrectomy. d OCT images of the right eye of a 57-year-old female. She has S1 MTM at baseline. The macular retinoschisis remained stable throughout the follow-up period

difference was found in the rate of resolving/stable or progressive MTM.

\section{Discussion}

In this study, patients' visual acuities and MS were significantly improved after cataract surgeries. Compared with the unoperated patients, cataract extraction did not increase the risk of MTM progression. Potential risk factors of MTM progression were the absence of PVD, stage 2-4 MTM at baseline and longer axial length.

Mechanisms of MTM include increasing axial length [8], progressive posterior staphyloma [26], and continuous vitreoretinal traction caused by incomplete PVD with vitreomacular adhesion [27], remnant posterior hyaloid [28], and epiretinal membrane [29]. Other causes were noncompliant internal limiting membrane (ILM) [30], vascular stiffness [31], scleral pathology [32], and microvascular abnormalities [33]. With continuity of these conditions, MTM can exacerbate to FTMH and foveal detachment, with remarkable visual impairment [6]. Vitrectomy, ILM peeling, and gas tamponade were useful procedures in restoring macular structures [10, 11]. However, macular surgery was not always necessary since in many cases MTM might not be sight-threatening [8, 22]. In some cases, MTM was even self-healing [15], needless of surgical repair. Attentions also should be paid to the possible sight-threatening complications after macular surgery $[12,13]$. Thus, vitrectomy was not definitely beneficial or necessary for patients with MTM, especially for those with minor MTM.

Incident cataract is another common disease causing visual impairment in highly myopic patients [1]. Simple cataract extraction might be effective in clearing the refracting media and improving visual acuity. Relationship of cataract surgery with MTM progression remained controversial. It was indicated that cataract extraction and lens implantation led to aggravation of MTM [16, 17, 18] and even induced retinal detachment [34, 35]. Lai et al. [19] reported that visual acuity improved after cataract extraction in patients with myopic foveoschisis, despite of the increase in central foveal thickness. Fourteen patients (20 eyes) were recruited and the mean followed up period was $15.6 \pm$ 9.6 months [19]. Although their study was retrospective, and the number of patients was limited without a control group, their efforts shed light on the feasibility of cataract surgery in patients with MTM by larger-scale controlled studies.

In this study, 179 highly myopic patients with MTM underwent cataract extraction and 50 other unoperated patients were enrolled as controls. All patients were followed up over 36 months. Almost all of the operated patients had significantly better BCVA and improved macular MS after surgeries except that one patient developed FTMH and foveal detachment and underwent vitreoretinal surgery (Fig. 3c). Notably, the mean BCVA of patients who and stable/improved MTM remained stable all through whole follow up, while the mean MS was slighted reduced after 3 years compared with that at 1 year after cataract surgery. A minority of subjects displayed better morphology on OCT images but did not show improvement in MS. The long-term swelling of optic nerve in myopic eyes may underlie the inconsistency between retinal morphology and function. Due to the long time the microperimetry examination takes, not all the unoperated patients were willing to complete the exam. And the cataract could have significant influence on the result of macular sensitivity exam. Thus, in this study, we performed the microperimetry examination only on patients who had underwent cataract surgeries.

We also evaluated the influences of cataract surgery on macular structures and found that $162(90.5 \%)$ patients had stable MTM. Four (2.2\%) patients even had resolution of MTM. Progressive MTM was found in $13(7.3 \%)$ operated patients and in $3(6.0 \%)$ unoperated patient. There was no difference in the rate of MTM progression between operated and unoperated patients $(p=0.757)$. We suggested that phacoemulsification with posterior chamber intraocular lens implantation did not add the risk of progressive MTM in highly myopic patients.

Possible mechanisms that cataract surgery caused fundus-threatening complications included disturbance to the vitreous body during surgery, increase of vitreoretinal traction by the posterior hyaloid, and PVD after surgery [28, 36, 37]. Continual improvements in phacoemulsification techniques and devices, however, increase the safety and efficiency of cataract surgeries [38, 39, 40]. Lens extraction with lower intraocular energy is realized with power modulations and supplemental energy sources. High level of vacuum for lens extraction reduces the need of ultrasound 
Fig. 4 The potential risk factors of MTM progression. a-f The comparison of age (a), axial length (b), diopter (c), nuclear opalescence (d), CDE (e), and US time (f) between stable/ improved and progressed groups shows that the average axial length of myopic eyes is significantly longer in the progressed group. $\mathbf{g}$ The $\chi^{2}$ test indicates that the larger extent of outer macular retinoschisis and negative PVD may confer higher risk for MTM progression a

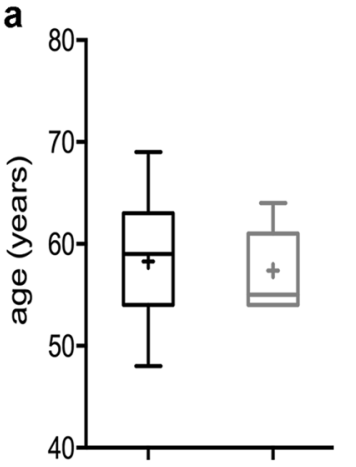

d

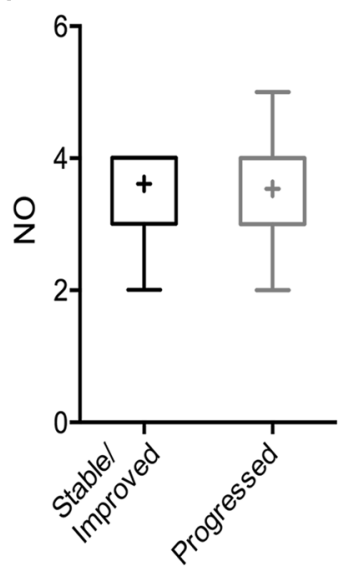

b

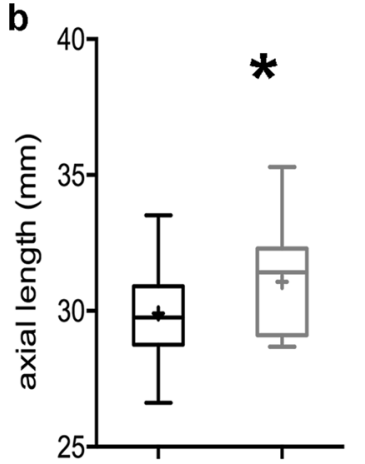

e

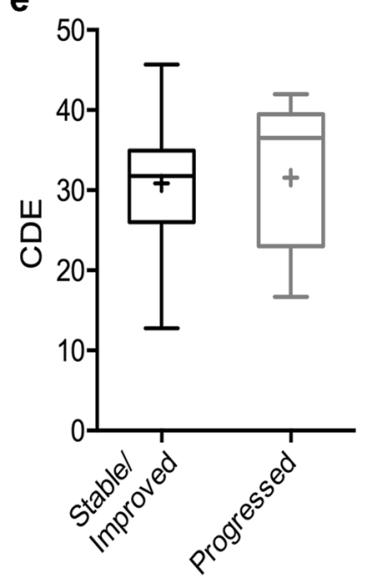

C

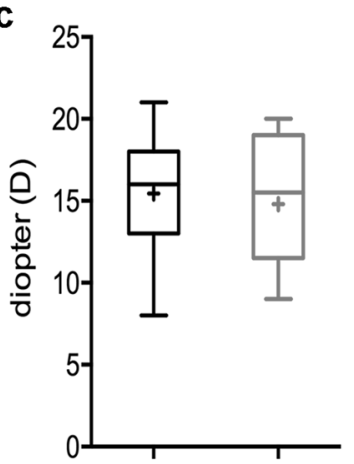

f

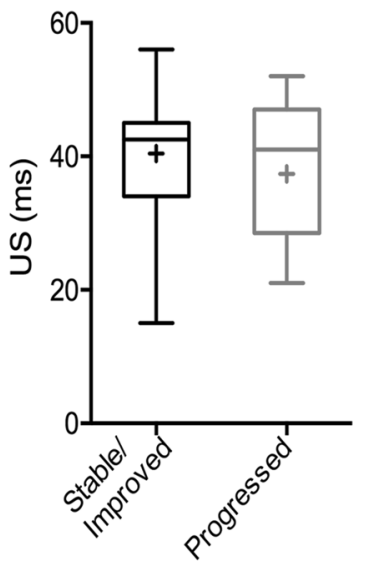

g

\begin{tabular}{|c|c|c|c|c|}
\hline & \multicolumn{2}{|c|}{ Outcome() } & \multirow[b]{2}{*}{$\begin{array}{c}\mathrm{p} \\
\text { Value }\end{array}$} & \multirow[b]{2}{*}{ Significance } \\
\hline & Stable/Improved & Progressed & & \\
\hline Gender & & & 0.407 & n.s \\
\hline male & 45.50 & 56.30 & & \\
\hline female & 54.50 & 43.80 & & \\
\hline PVD & & & & \\
\hline positive & 37.10 & 68.80 & 0.012 & * \\
\hline negative & 62.90 & 31.30 & & \\
\hline Grouping & & & 0.003 & * \\
\hline SO & 17.80 & 0.00 & & \\
\hline S1 & 23.50 & 0.00 & & \\
\hline S2 & 17.80 & 18.80 & & \\
\hline S3 & 24.40 & 31.30 & & \\
\hline S4 & 16.40 & 50.00 & & \\
\hline $\begin{array}{l}\text { Cataract } \\
\text { surgery }\end{array}$ & & & .757 & n.s \\
\hline positive & 22.10 & 18.80 & & \\
\hline negative & 77.90 & 81.30 & & \\
\hline
\end{tabular}

energy for emulsification, prevents complications such as iris and corneal damages, and improves the prognosis of visual acuities. Smaller corneal incision improves fluidics, and thus provides guarantee for more stable anterior chamber and less disturbance to the vitreous body.

Our results showed that preexisting vitreomacular adhesion (no PVD) and MTM stage 2-4 were risk factors of long-term MTM progression. These findings were also suggested in previous studies [8, 27]. In operated eyes, complete PVD prior to cataract surgery may reduce abnormal vitreoretinal traction caused by intraoperative vitreous disturbance and prevent the further progression of MTM. In unoperated eyes, preexisting PVD eliminates additional vitreoretinal traction induced by subsequent elongation of the axial length $[8,26]$. Shimada et al. [20] reported that eyes with more extensive macular retinoschisis tended to progress more commonly. They suggested that patients with severe MTM should be followed more 
frequently in case that MTM progressed and visual acuity decreased. In our study, patients with S2, S3, and S4 MTM (not S4 alone) tended to progress more commonly than those with S0 and S1 MTM. One operated patient with S4 MTM even underwent vitrectomy for FTMH and foveal detachment (Fig. 3c). No differences were found in the rate of progressive MTM among S2, S3, and S4 MTM. Hence, we recommend that patients with S2 to S4 MTM should be routinely followed after cataract surgeries for at least 3 years. It is noticed that, MTM progressed in most of the patients later than 6 months after cataract surgery. In that case, it may not be the cataract surgery which caused the progression of MTA, but the natural cause of the maculopathy. The patient who developed FTMH in 6 months after surgery had S3 MTM and experienced a significant decrease in visual acuity soon after strenuous activity. It is suggested that close attention should be payed to people who got sever stage of MTM and vigorous exercise might better be avoided in 6 months after surgery.

Taken together, our study reveals that cataract surgery generally improves the visual acuity and macular sensitivity of highly myopic patients with MTM. Phacoemulsification and IOL implantation in highly myopic patients do not increase the risk of progressive MTM. Preoperative vitreoretinal adhesion, longer axial length, and S2 to S4 MTM are risk factors for progressive MTM. A long-term follow-up on the development of MTM is recommended.

\section{Summary}

\section{What was known before}

- High myopia increase the risk for MTM and early development of cataracts; cataract extraction improves visual acuity in highly myopia patients; cataract extraction increases central foveal thickness in patients with myopic foveoschisis.

\section{What this study adds}

- MTM remains stable in a majority of subjects after cataract surgery; long AL, incomplete PVD, and entire macular area retinoschisis at initial evaluation may confer high risk for the progress of MTM after cataract surgery.

Acknowledgements This work was supported by grants National Natural Science Foundation of China (No. 81670835 and No. 81700851). The study is registered with the identifier NCT03062085 at ClinicalTrials.gov.

\section{Compliance with ethical standards}

Conflict of interest The authors declare that they have no conflict of interest.

Publisher's note: Springer Nature remains neutral with regard to jurisdictional claims in published maps and institutional affiliations.

\section{References}

1. Xu L, Li J, Cui T, Hu A, Fan G, Zhang R, et al. Refractive error in urban and rural adult Chinese in Beijing. Ophthalmology. 2005;112:1676-83.

2. Iwase A, Araie M, Tomidokoro A, Yamamoto T, Shimizu H, Kitazawa $\mathrm{Y}$, et al. Prevalence and causes of low vision and blindness in a Japanese adult population: the Tajimi Study. Ophthalmology. 2006;113:1354-62.

3. Friedman NJ, Kaiser PK. Essentials of Ophthalmology. Philadelphia, PA: Elsevier Inc.; 2007.

4. Ikuno Y. Overview of the complications of high myopia. Retina. 2017;37:2347-51.

5. Pan CW, Cheng CY, Saw SM, Wang JJ, Wong TY. Myopia and age-related cataract: a systematic review and meta-analysis. Am J Ophthalmol. 2013;156:1021-33. e1021.

6. Panozzo G, Mercanti A. Optical coherence tomography findings in myopic traction maculopathy. Arch Ophthalmol. 2004;122:1455-60.

7. Takano M, Kishi S. Foveal retinoschisis and retinal detachment in severely myopic eyes with posterior staphyloma. Am J Ophthalmol. 1999;128:472-6.

8. Baba T, Ohno-Matsui K, Futagami S, Yoshida T, Yasuzumi K, Kojima A, et al. Prevalence and characteristics of foveal retinal detachment without macular hole in high myopia. Am J Ophthalmol. 2003;135:338-42.

9. Ouyang PB, Duan XC, Zhu XH. Diagnosis and treatment of myopic traction maculopathy. Int J Ophthalmol. 2012;5:754-8.

10. Kobayashi H, Kishi S. Vitreous surgery for highly myopic eyes with foveal detachment and retinoschisis. Ophthalmology. 2003;110:1702-7.

11. Panozzo G, Mercanti A. Vitrectomy for myopic traction maculopathy. Arch Ophthalmol. 2007;125:767-72.

12. Hirakata A, Hida T. Vitrectomy for myopic posterior retinoschisis or foveal detachment. Jpn J Ophthalmol. 2006;50:53-61.

13. Ikuno Y, Tano Y. Early macular holes with retinoschisis in highly myopic eyes. Am J Ophthalmol. 2003;136:741-4.

14. Hotta K, Hotta J. Retinoschisis with macular retinal detachment associated with vitreomacular traction syndrome. Retina. 2004;24:307-9.

15. Polito A, Lanzetta P, Del Borrello M, Bandello F. Spontaneous resolution of a shallow detachment of the macula in a highly myopic eye. Am J Ophthalmol. 2003;135:546-7.

16. Haug SJ, Bhisitkul RB. Risk factors for retinal detachment following cataract surgery. Curr Opin Ophthalmol. 2012;23:7-11.

17. Ivastinovic D, Schwab C, Borkenstein A, Lackner EM, Wedrich A, Velikay-Parel M. Evolution of early changes at the vitreoretinal interface after cataract surgery determined by optical coherence tomography and ultrasonography. Am J Ophthalmol. 2012;153:705-9.

18. Petousis V, Sallam AA, Haynes RJ, Patel CK, Tyagi AK, Kirkpatrick JN, et al. Risk factors for retinal detachment following cataract surgery: the impact of posterior capsular rupture. $\mathrm{Br} \mathbf{J}$ Ophthalmol. 2016;100:1461-5. 
19. Lai CC, Yeung L, Chen YP, Wang NK, Wu WC, Chen KJ, et al. Macular and visual outcomes after cataract extraction for highly myopic foveoschisis. J Cataract Refract Surg. 2008; 34:1152-6.

20. Shimada N, Tanaka Y, Tokoro T, Ohno-Matsui K. Natural course of myopic traction maculopathy and factors associated with progression or resolution. Am J Ophthalmol. 2013;156:948-57. e941.

21. Xu L, Li Y, Wang S, Wang Y, Wang Y, Jonas JB. Characteristics of highly myopic eyes: the Beijing Eye Study. Ophthalmology. 2007;114:121-6.

22. Smiddy WE, Kim SS, Lujan BJ, Gregori G. Myopic traction maculopathy: spectral domain optical coherence tomographic imaging and a hypothesized mechanism. Ophthalmic Surg Lasers Imaging. 2009;40:169-73.

23. Sun Z, Gao H, Wang M, Chang Q, Xu G. Rapid progression of foveomacular retinoschisis in young myopics. Retina. 2018. https:// doi.org/10.1097/IAE.0000000000002203 [Epub ahead of print].

24. Midena E, Radin PP, Pilotto E, Ghirlando A, Convento E, Varano $M$. Fixation pattern and macular sensitivity in eyes with subfoveal choroidal neovascularization secondary to age-related macular degeneration. A microperimetry study. Semin Ophthalmol. 2004;19:55-61.

25. Li YK, Yang JM, Huang YB, Ren DD, Chi FL. Shrinkage of ipsilateral taste buds and hyperplasia of contralateral taste buds following chorda tympani nerve transection. Neural Regen Res. 2015;10:989-95.

26. VanderBeek BL, Johnson MW. The diversity of traction mechanisms in myopic traction maculopathy. Am J Ophthalmol. 2012;153:93-102.

27. Gaucher D, Haouchine B, Tadayoni R, Massin P, Erginay A, Benhamou N, et al. Long-term follow-up of high myopic foveoschisis: natural course and surgical outcome. Am J Ophthalmol. 2007;143:455-62.

28. Spaide RF, Fisher Y. Removal of adherent cortical vitreous plaques without removing the internal limiting membrane in the repair of macular detachments in highly myopic eyes. Retina. 2005;25:290-5.
29. Sayanagi K, Morimoto Y, Ikuno Y, Tano Y. Spectral-domain optical coherence tomographic findings in myopic foveoschisis. Retina. 2010;30:623-8.

30. Kumagai K, Furukawa M, Ogino N, Larson E. Factors correlated with postoperative visual acuity after vitrectomy and internal limiting membrane peeling for myopic foveoschisis. Retina. 2010;30:874-80.

31. Ikuno Y, Gomi F, Tano Y. Potent retinal arteriolar traction as a possible cause of myopic foveoschisis. Am J Ophthalmol. 2005;139:462-7.

32. Rada JA, Shelton S, Norton TT. The sclera and myopia. Exp Eye Res. 2006;82:185-200.

33. Hayashi W, Shimada N, Hayashi K, Moriyama M, Yoshida T, Tokoro T, et al. Retinal vessels and high myopia. Ophthalmology. 2011;118:791-91. e792.

34. Ripandelli G, Scassa C, Parisi V, Gazzaniga D, D’Amico DJ, Stirpe M. Cataract surgery as a risk factor for retinal detachment in very highly myopic eyes. Ophthalmology. 2003;110:2355-61.

35. Ripandelli G, Coppe AM, Parisi V, Olzi D, Scassa C, Chiaravalloti A, et al. Posterior vitreous detachment and retinal detachment after cataract surgery. Ophthalmology. 2007;114:692-7.

36. Neal RE, Bettelheim FA, Lin C, Winn KC, Garland DL, Zigler JS, et al. Alterations in human vitreous humour following cataract extraction. Exp Eye Res. 2005;80:337-47.

37. Sakaguchi H, Ikuno Y, Choi JS, Ohji M, Tano T. Multiple components of epiretinal tissues detected by triamcinolone and indocyanine green in macular hole and retinal detachment as a result of high myopia. Am J Ophthalmol. 2004;138:1079-81.

38. Clark A, Morlet N, Ng JQ, Preen DB, Semmens JB. Risk for retinal detachment after phacoemulsification: a whole-population study of cataract surgery outcomes. Arch Ophthalmol. 2012; 130:882-8.

39. Hoffman RS, Fine IH, Packer M. New phacoemulsification technology. Curr Opin Ophthalmol. 2005;16:38-43.

40. Fine IH, Packer M, Hoffman RS. Power modulations in new phacoemulsification technology: improved outcomes. J Cataract Refract Surg. 2004;30:1014-9. 